\title{
ARTICLE
}

\section{Radiation shielding in SPring-8 linear accelerator tunnels for safety interlock system upgrade}

\author{
Nobuteru Nariyama* \\ Japan Synchrotron Radiation Research Institute, 1-1-1, Kouto, Sayo, Hyogo 679-5198, Japan
}

\begin{abstract}
To upgrade a safety interlock system, a restricted area was converted into an accessible area at an operating condition. To judge the necessity for an additional shield design, $\gamma$-ray and neutron dose distributions were measured in the linac, booster synchrotron, L3BT, and New SUBARU (NS) areas. In the linac, chicane was found to be the main source of $\gamma$ rays. Among the new accessible areas, the $\gamma$-ray doses exceeded the limit at the electron transport openings of the synchrotron and NS areas. Similarly, the neutron dose surpassed the limit at the synchrotron opening. The beam-dump contribution to the neutron dose was estimated to be $20 \%$ by using the Monte Carlo code FLUKA.
\end{abstract}

\section{Keywords: electron linear accelerator; local shield; dose; Monte Carlo; FLUKA; dump; streaming}

\section{Introduction}

The earlier SPring-8 accelerator safety interlock system consisted of an operating mode system and four accelerator interlock systems of linac (Li)-booster synchrotron (Sy), L3, storage ring (SR), and New SUBARU (NS) [1]. An operating mode such as a storage or top up is input into the operating mode system, which permits gun and RF operation in each area at the selected mode. In this context, the system is based on the operating mode.

The increasing number of operation modes, however, has complicated the operating system. Moreover, the once-expected addition of a safety interlock system from an X-ray free-electron laser facility (SACLA) [2, 3] to the SPring- 8 interlock system possibly complicated the system further. To avoid these future problems, a new system was designed to permit area-by-area gun and RF operation, independent of the operating mode [1]. In this system, a new area could be added without existing area interference. Five areas comprising Li, Sy, L3, SR, and NS were defined. Access to any area where the electron transport and accumulation were not permitted had to be possible. As a result, when electrons were injected only into the linac L2 dump, the synchrotron and L3 areas, which were connected to the linac area through the transport pipes as shown in Figure 1, were assumed not to be a radiation hazard for the personnel inside; this design was different from the previous one. Similarly, when the electrons were injected only into the L3 dump, the dose in the neighboring NS area, as shown in Figure 2, had to be lower than a limit. This meant that when the dose in the new accessible area exceeded the limit, local

*Corresponding author. Email: nariyama@spring8.or.jp shields became necessary. For an effective design to be possible, the source conditions had to be clarified.

In this study, to obtain the source information, $\gamma$-ray and neutron dose distributions in the linac tunnels were measured before the upgrade. If the dump influence became clear, the influence from other sources would also become clear. The contribution from the beam dump was calculated with a Monte Carlo code.

\section{Measurements in the accelerator tunnels}

\subsection{Materials and methods}

The $\gamma$-ray and neutron dose distributions were measured in the linac, Sy, L3, and NS areas. The electrons were incident onto the L2 dump in two cases. In the second case, the electrons lost energy at the chicane for 15 min because of an unintended operation. In the third case, the electrons were incident onto the L3 dump. The electron energy was $1 \mathrm{GeV}$. The numbers for the L2 dump were $3.35 \times 10^{15}(536 \mu \mathrm{C})$ and $5.11 \times 10^{15}$ $(819 \mu \mathrm{C})$, and the number for the L3 dump was $1.75 \times$ $10^{15}(280 \mu \mathrm{C})$ at $10 \mathrm{pps}$ with 40 -nsec pulse width.

Direct ion storage dosimeters (DIS), thermoluminescent dosimeters (TLD, LiF:Mg, Cu,P), and optically stimulated luminescent dosimeters (OSLD, $\mathrm{Al}_{2} \mathrm{O}_{3}: \mathrm{C}$ ) were used at almost all the points in the new accessible areas. The applicable dose limits were 1, 5 and $10 \mu \mathrm{Sv}$, respectively. For neutrons, rem counters (Aloka) were set in the new accessible areas, and solid-state track detectors (CR-39) and bubble detectors (Bubble Technology Industries) were used around the exit of the transport holes for the small sizes. The dose limit of CR-39 was $200 \mu \mathrm{Sv}$ and the sensitivities of the 
bubble detectors were 3 bubbles/ $\mu$ Sv. In the beam area, OSLDs and CR-39 were set to deal with the intense doses. The dosimeters were calibrated with ${ }^{137} \mathrm{Cs}$ and ${ }^{60} \mathrm{Co}$ sources for $\gamma$ rays and ${ }^{252} \mathrm{Cf}$ and ${ }^{241} \mathrm{Am}$-Be sources for neutrons.

The duct diameter for electron transport was $25 \mathrm{~cm}$ for Sy and NS. The offset duct to L3 was 30 and $50 \mathrm{~cm}$ in diameter. The lengths were $2 \mathrm{~m}$ for Sy, $3.4 \mathrm{~m}$ for L3, and $3 \mathrm{~m}$ for NS.

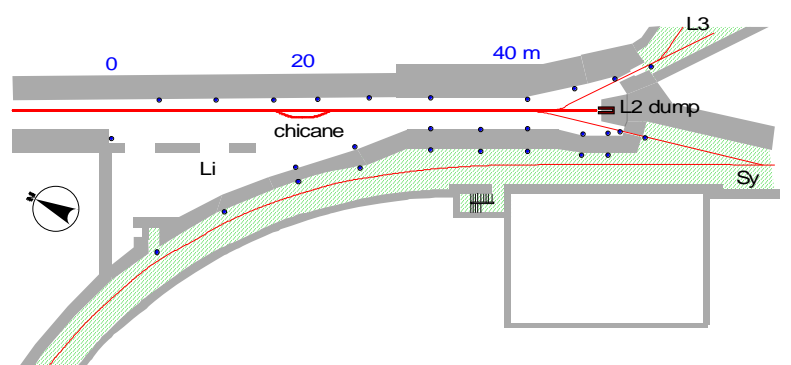

Figure 1. Linac area. Red line denotes the electron beamline. $1-\mathrm{GeV}$ electrons were incident onto the L2 dump. Sy and L3 areas, which are green hatched, were the new accessible areas for this operation. Blue dots denote the dosimeter positions. Distance in meters is used in Figure 3.

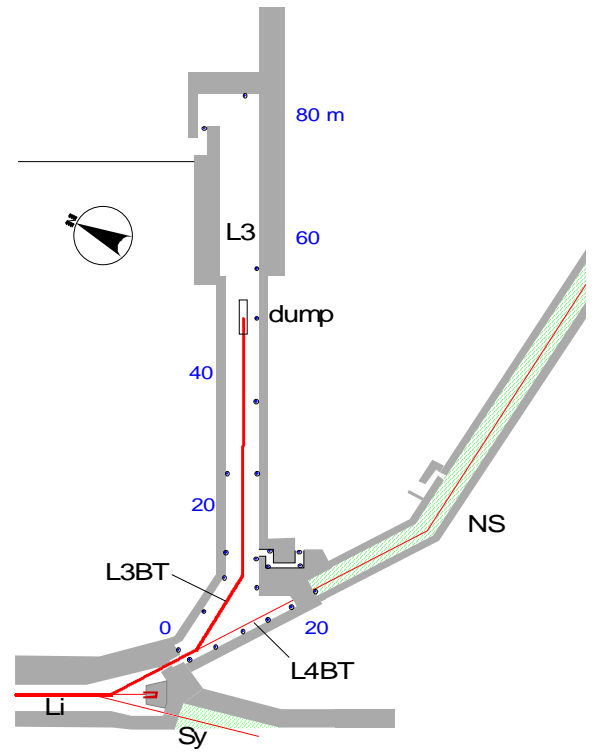

Figure 2. L3 area. 1-GeV electrons were incident onto the L3 dump from the linac. NS area is the new accessible area. Distance in meters is used in Figure 4.

\subsection{Results}

\subsubsection{Source information}

Figure 3 shows the result of the $\gamma$-ray and neutron doses in the linac area. The position expresses the distance from the north wall as shown in Figure 1. The doses were converted into doses per week by using the permitted electron numbers incident onto L2 and L3 dumps per week of $5.7 \times 10^{16}$ and $3.7 \times 10^{15}$. The readings of three kinds of dosimeters for $\gamma$ rays were used as follows: DIS below $5 \mu \mathrm{Sv}$, TLDs at $5-10 \mu \mathrm{Sv}$ and OSLDs above $10 \mu \mathrm{Sv}$. At the exit of the hole in Sy, the value of CR-39 became available for the neutron dose. On the other hand, the value of bubble detectors was used in L3 because of the small dose level.

The $\gamma$-ray doses increased from the chicane to the $30-\mathrm{m}$ position and gradually decreased toward the dump. For the second measurement, the dose increased considerably at the position diagonally forward from the chicane. On the other hand, the neutron dose exhibited peaks near the chicane and the dump; the neutron doses showed the source positions more clearly. From these results, it was concluded that the chicane was the main $\gamma$-ray source, and the neutron sources were estimated to be located at the chicane and the dump.

The doses in the new accessible area are also indicated in Figure 3. At the beam transport opening of Sy, both $\gamma$-ray and neutron doses exceeded the limit of 1 $\mathrm{mSv}$ per week. In L3, neither exceeded the limit.
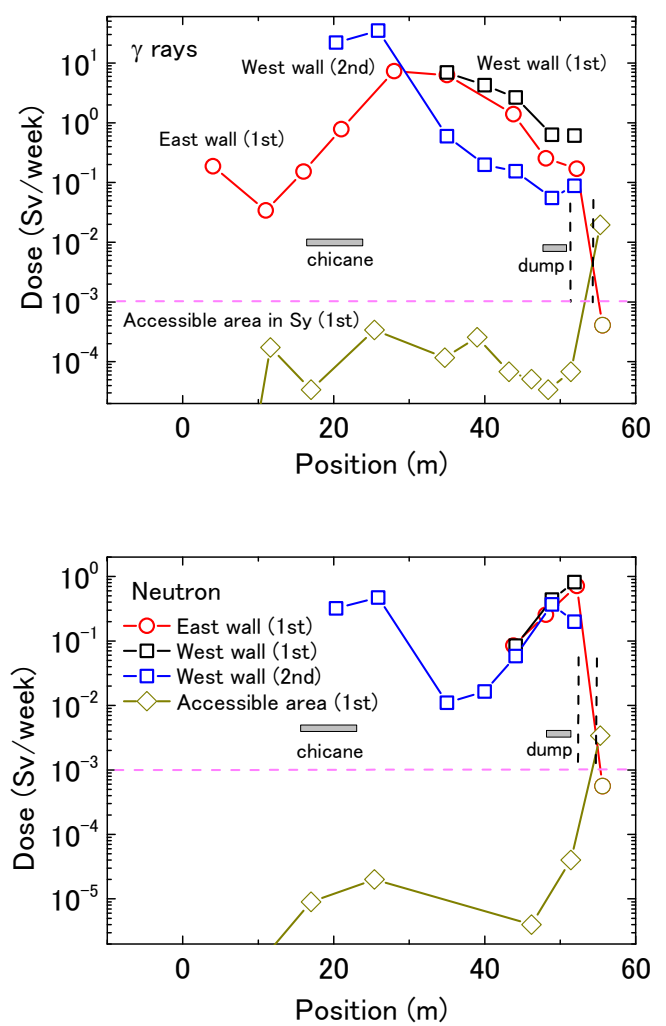

Figure 3. First and second measured $\gamma$-ray and neutron dose distributions on the linac wall. The position denotes the distance from the northwest wall. Dashed lines denote the concrete with holes. Doses are converted into doses per week, of which the limit for access is expressed as the red dotted line.

Figure 4 shows the result for the L3 area. The position expresses the distance from the linac opening. The $\gamma$-ray doses showed maxima in the upstream region and decreased by one order of magnitude toward the corner. The value almost remained constant between the corner and the dump. Along the L4BT wall, the doses decreased steeply. This showed that intense sources did not exist along the L4BT. The neutron doses were one 
order of magnitude smaller than the $\gamma$-ray doses. These results showed that the $\gamma$-ray and neutron sources appeared to be situated downstream of the linac switching magnet and that weak sources existed around the bending magnet at the L3 corner. In the new accessible area of NS, the $\gamma$-ray dose exceeded the limit.

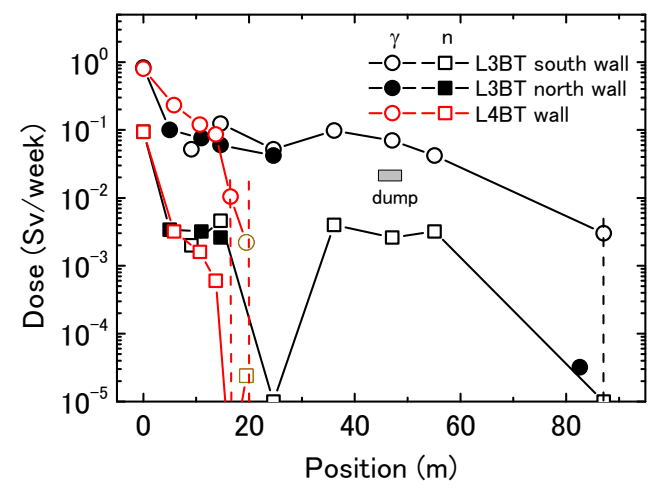

Figure 4. Measured $\gamma$-ray and neutron dose distribution along $\mathrm{L} 3 \mathrm{BT}$ and L4BT. The position denotes the distance from the upstream bottom. Red broken lines denote 3.4-m-thick concrete with hole on L4BT. Black broken lines represent the wall at the end of the L3 tunnel.

\subsubsection{Addition of local shielding}

The measured doses exceeded the limit at two locations in the accessible areas: the $\gamma$-ray and neutron doses at the transport opening to Sy, and the $\gamma$-ray doses at the transport opening to NS. The linac measurement data suggested that the L2 dump neutrons influenced the dose in Sy through the duct. Moreover, the chicane photons inevitably entered the duct to Sy because of the geometry, so that streaming component suppression became important. Because the experimental result was only 20 times larger than the limit, only narrowing the sight from the chicane to the entrance of the duct was considered to lower the dose at the duct exit below the limit.

As a result, 10-cm-thick polyethylene was set around the duct entrance to decrease the neutron component from the dump, and 10-cm-thick polyethylene and 16-cm-thick lead were set $1 \mathrm{~m}$ upstream from the position for the chicane also considering the loss scenario. Moreover, lead and polyethylene beans were stuffed in the gap between the 9-cm-diameter transport pipe and the concrete holes. As a result, the doses decreased to $65 \mu \mathrm{Sv}$ and $14 \mu \mathrm{Sv}$ per rated output for $\gamma$-ray and neutron doses at the Sy exit.

In front of the L4BT hole, a 12-cm-thick lead shield was set also considering the electron loss along L3BT, and it lowered the dose to an undetectable level. The pipe of L4 was an oval of height $4.5 \mathrm{~cm}$. In the pipes to Sy and L4, radiation shutters were located just downstream from the fork in the $\mathrm{Li}$ area, which obstructed the dark current through the pipes.

\section{Analysis of the influence of the L2 beam dump}

From the measurements, the chicane and dump were found to be the dominant sources in the linac. While the analysis of the chicane radiation was difficult, it was possible to analyze the dump. Because the estimation was expected to make each contribution clear, the $\gamma$-ray and neutron dose distributions near the L2 dump were calculated with the Monte Carlo code FLUKA (Ver. 2011.2.11) [4] with the FLAIR user interface on a 64-bit Fedora16. Parallel calculations were executed on four cores of an Intel Xeon CPU.
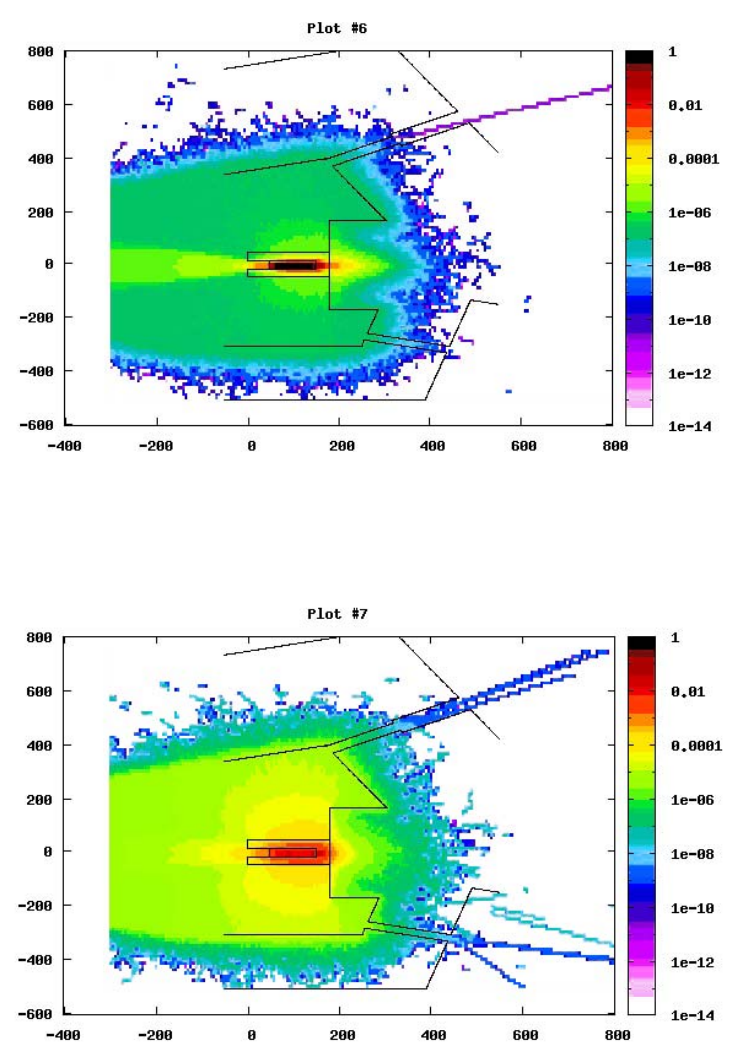

Figure 5. $\gamma$-ray (top) and neutron (bottom) dose distributions calculated with FLUKA around the L2 dump. Contour units are in $\mathrm{pSv} / \mathrm{e}^{-}$and " $1 \mathrm{e}-14$ " is read as " $1 \times 10^{-14}$ ". The scales are in centimeter. Particles were integrated within the width equal to the diameter of the Sy duct centering on the duct height.

Figure 5 shows the results of the $\gamma$-ray and neutron doses in the linac around the dump. The dump was composed of a graphite cylinder of $30-\mathrm{cm}$ diameter and $100-\mathrm{cm}$ length in the hollow of the iron cylinder of $90-\mathrm{cm}$ diameter. Compared with the neutrons, the photons easily proceed forward and backward, so that the photons scarcely penetrated the openings. On the other hand, the neutrons scattered in all directions and contributed $20 \%$ and $33 \%$ to the neutron doses at the Sy and L3 openings, respectively. 
Figure 6 shows the neutron dose distribution on both sides of the L2 dump, which is a cross-sectional view of Figure 5. The doses on the east and west walls were found to be $1.2 \times 10^{-5}$ and $1.8 \times 10^{-5} \mathrm{pSv} / \mathrm{e}^{-}$, which corresponded to 0.68 and $1.0 \mathrm{~Sv} /$ week. The doses almost agreed with the measured values of 0.71 and 0.81 $\mathrm{Sv} /$ week as shown in Figure 3.

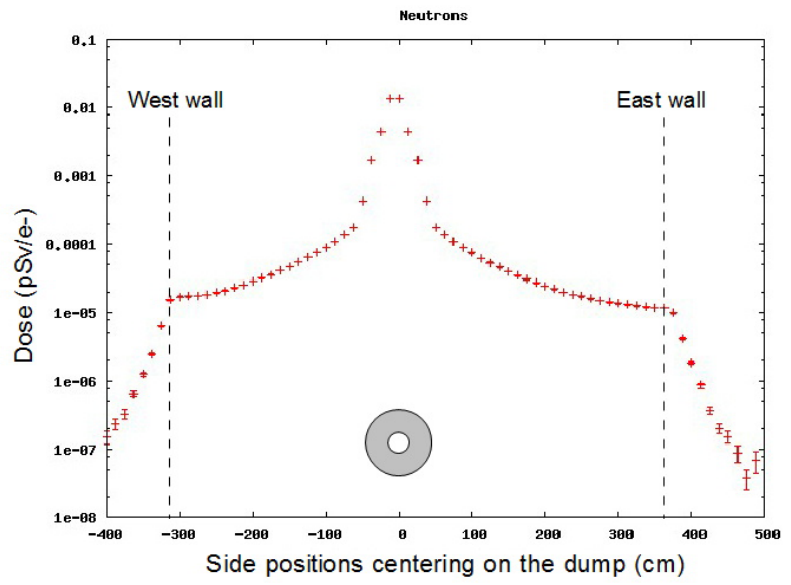

Figure 6. Neutron dose distribution on both sides of the L2 dump in Figure 5. The error bars express the statistical fluctuations.

\section{Conclusion}

In the present study, the geometry was severe because the ducts were situated in the forward direction of the main source. The chicane-source intensity could not be estimated analytically; the measurement was an effective tool to set the local shields. For unintended operations, another local lead shield was set downstream of the chicane after the confirmation measurement.

The Monte Carlo calculation, which was performed after the shield setting, was helpful to understand the measurement results, especially streaming components, because it provided information other than that obtained by measurement concerning the dump contribution.

\section{Acknowledgements}

The author would like to thank H. Dewa and S. Suzuki of JASRI for the linac operation. We also thank M. Okita and Y. Tsuzuki for their assistance with the radiation measurements.

\section{References}

[1] C. Saji, M. Toko, T. Matsushita, et al., Upgrade of accelerator radiation safety system for SPring-8 (2), Proc. 8th Ann. Meet. Part. Accel. Soc. Japan, Tsukuba, Japan, 2011, (2011), pp. 547-550. [in Japanese]

[2] N. Nariyama, T. Matsushita, H. Aoyagi, M. Kago, T.Itoga, C. Saji, R. Tanaka and Y. Asano, Concept of radiation monitoring and safety interlock systems for XFEL/SPring-8, Proc. IPAC'10, Kyoto, Japan, 2010, (2010), pp. 2836-2838.

[3] T. Ishikawa, H. Aoyagi, T. Asaka, et al., A compact X-ray free-electron laser emitting in the sub-ångström region, Nat. Photonics, 6 (2012), pp. 540-544.

[4] A. Ferrari, P.R. Sala, A. Fassò and J. Ranft, FLUKA: A Multi-particle Transport Code, CERN2005-10 (2005), INFN/TC_05/11, SLAC-R-773. 